\title{
A GENERALIZATION OF A RESULT FOR ITERATIVE FUNCTIONS IN $\mathbb{R}^{n}$
}

\author{
Béla FINTA \\ University of Medicine, Pharmacy, Sciences and Technology of Tîrgu Mures \\ Gheorghe Marinescu Street, no. 38, 540139 Tîrgu Mureş, Romania \\ e-mail: bela.finta@umfst.ro
}

\begin{abstract}
The purpose of this paper is to generalize for real vector-valued functions of a result for real iterative functions and to give some applications for nonlinear system of equations.
\end{abstract}

Keywords: nonlinear equations, nonlinear system of equations, Banach fixed point theorem, mean value theorem for real vector-valued functions

\section{Introduction}

It is known the following result for iterative functions on the real line, see for example [?] or [?]

Theorem 1. ( general theorem for real iterative functions) If $\phi: J \rightarrow \mathbb{R}$ is derivable on the interval $J=\left[x_{0}-\delta, x_{0}+\delta\right], \delta>0$ and the derivative function $\phi^{\prime}$ satisfies the inequality $0 \leq\left|\phi^{\prime}(x)\right| \leq m<1$ for every $x \in J$ and the point $x_{1}=\phi\left(x_{0}\right)$ verifies the inequality $\left|x_{1}-x_{0}\right| \leq(1-m) \delta$, then:

- we can form the sequence $\left\{x_{k}\right\}_{k \in \mathbb{N}}$ with the iterative rule $x_{k+1}=\phi\left(x_{k}\right), k \in \mathbb{N}$, such that for every $k \in \mathbb{N}$ we have $x_{k} \in J$;

- there exists $\lim _{k \rightarrow \infty} x_{k}=x^{*} \in J$;

- $x^{*}$ is the unique solution of the equation $\phi(x)=$ $x$ in the interval $J$.

In [?] we showed a complex variant of this theorem using [?] and [?]:

Theorem 2. ( general theorem for complex iterative functions ) If $\phi: B\left(z_{0}, r\right) \rightarrow \mathbb{C}$ is a holomorphic function on the closed disc $B\left(z_{0}, r\right) \subset \mathbb{C}, z_{0} \in \mathbb{C}, r>0$, such that the derivative function $\phi^{\prime}$ satisfies the inequality $0 \leq\left|\phi^{\prime}(z)\right| \leq m<\frac{\sqrt{2}}{2}$ for every $z \in$
$B\left(z_{0}, r\right)$ and the point $z_{1}=\phi\left(z_{0}\right)$ verifies the inequality $\left|z_{1}-z_{0}\right| \leq(1-\sqrt{2} \cdot m) \cdot r$, then:

- we can form the sequence $\left\{z_{k}\right\}_{k \in \mathbb{N}}$ with the iterative rule $z_{k+1}=\phi\left(z_{k}\right), k \in \mathbb{N}$, such that for every $k \in \mathbb{N}$ we have $z_{k} \in B\left(z_{0}, r\right)$;

- there exists the limit of the sequence $\left\{z_{k}\right\}_{k \in \mathbb{N}}$ and $\lim _{k \rightarrow \infty} z_{k}=z^{*} \in B\left(z_{0}, r\right)$;

- $z^{*}$ is the unique solution of the equation $\phi(z)=z$ in the closed disc $B\left(z_{0}, r\right)$.

Using theorem 2 in [?] we gave some applications for nonlinear complex equations. In order to solve the complex equation $f(z)=0$ we used the following transformations: translation, translation and homothety, the Newton's method in complex case and we builded the complex version of parallel, chord and Steffensen's method.

The purpose of this paper is to show an extension of these theorems in the space $\mathbb{R}^{n}$.

(C) 2019 Published by University Press. This is an open access article licensed under the Creative Commons AttributionNonCommercial-NoDerivs License (http://creativecommons.org/ licenses/by-nc-nd/3.0/). 


\section{Main part}

Let us consider the euclidian norm $\|\cdot\|$ on the space $\mathbb{R}^{n}$ and the closed sphere $B(w, r)=\{x \in$ $\left.\mathbb{R}^{n} /\|x-w\| \leq r\right\}$ in the space $\mathbb{R}^{n}$, with center $w$ and radius $r>0$. First we remember the Banach fixed point theorem in the case of the closed sphere $B(w, r)$ :

Theorem 3. Let $\phi: B(w, r) \rightarrow B(w, r)$ be a contraction, i.e. there exists the constant $\alpha \in[0,1)$ such that $\|\phi(x)-\phi(y)\| \leq \alpha \cdot\|x-y\|$ for every $x, y \in B(w, r)$. Then the function $\phi$ has a unique fixed point in $B(w, r)$, which can be obtained as the limit of the sequence $\left\{x^{k}\right\}_{k \in \mathbb{N}}$ given by the iteration $x^{k+1}=\phi\left(x^{k}\right), k \in \mathbb{N}$, for every $x^{0} \in B(w, r)$.

Proof. Because $B(w, r) \subset \mathbb{R}^{n}$ is a closed sphere in the space $\mathbb{R}^{n}$, will be a Banach space, too. Now we apply the Banach fixed point theorem for the function $\phi: B(w, r) \rightarrow B(w, r)$.

We say that the function $\phi: B(w, r) \rightarrow \mathbb{R}^{n}$ is differentiable function on the closed sphere $B(w, r)$, if it is differentiable in every point $x \in B(w, r)$. If the point $x$ is a boundary point of the closed sphere $B(w, r)$, then we suppose that the function $\phi$ is defined on a small open disc with center $x$ and it is differentiable in $x$.

Theorem 4. Let $\phi: B(w, r) \rightarrow \mathbb{R}^{n}$ be a continuously differentiable function on the closed sphere $B(w, r)$, and $x \in B(w, r), h \in \mathbb{R}^{n}$ such that $x+h \in B(w, r)$. If there exists $M \geq 0$ positive real number such that for every $u \in[x, x+h]=\{x+t \cdot h / t \in[0,1]\}$ (the line segment $[x, x+h] \subset \mathbb{R}^{n}$ with endpoints $x$ and $x+h$ ) we have $\|D(\phi)(u)\| \leq M$, then $\|\phi(x+h)-\phi(x)\| \leq$ $M \cdot\|h\|$.

Proof. See for example [?].

Theorem 5. If the euclidian norm of $D(\phi)(u)$ is bounded on the closed disc $B(w, r) \subset \mathbb{R}^{n}$, i.e. there exists $M>0$ real number such that $\|D(\phi)(u)\| \leq M$ for every $u \in B(w, r)$, then $\|\phi(x)-\phi(y)\| \leq M$. $\|x-y\|$ for every $x, y \in B(w, r)$.

Proof. It is immediately from the previous theorem 4.

Theorem 6. ( general theorem for real $n$ dimensional iterative functions) If $\phi: B\left(x^{0}, r\right) \rightarrow \mathbb{R}^{n}$ is a continuously differentiable function on the closed sphere $B\left(x^{0}, r\right), x^{0} \in \mathbb{R}^{n}, r>0$, such that the differential function $D(\phi)$ satisfies the inequality $0 \leq$ $\|D(\phi)(u)\| \leq M<1$ for every $u \in B\left(x^{0}, r\right)$ and the point $x^{1}=\phi\left(x^{0}\right)$ verifies the inequality $\left\|x^{1}-x^{0}\right\| \leq$ $(1-M) \cdot r$, then:

- we can form the sequence $\left\{x^{k}\right\}_{k \in \mathbb{N}}$ with the iterative rule $x^{k+1}=\phi\left(x^{k}\right), k \in \mathbb{N}$, such that for every $k \in \mathbb{N}$ we have $x^{k} \in B\left(x^{0}, r\right)$;
- there exists the limit of the sequence $\left\{x^{k}\right\}_{k \in \mathbb{N}}$ and $\lim _{k \rightarrow \infty} x^{k}=x^{*} \in B\left(x^{0}, r\right)$;

- $x^{*}$ is the unique solution of the equation $\phi(x)=$ $x$ in the closed sphere $B\left(x^{0}, r\right)$.

Proof. Using theorem 5 for every $x, y \in B\left(x^{0}, r\right)$ we get $\|\phi(x)-\phi(y)\| \leq M \cdot\|x-y\|$ with $M<1$, so $\phi$ is a contraction. Also we obtain for every $x \in B\left(x^{0}, r\right)$ that $\left\|\phi(x)-\phi\left(x^{0}\right)\right\| \leq M \cdot\left\|x-x^{0}\right\|$. Now we show that $\phi\left(B\left(x^{0}, r\right)\right) \subset B\left(x^{0}, r\right)$, i.e. $\phi: B\left(x^{0}, r\right) \rightarrow$ $B\left(x^{0}, r\right)$. Indeed, for every $x \in B\left(x^{0}, r\right)$ we get $\left\|\phi(x)-x^{0}\right\|=\left\|\phi(x)-\phi\left(x^{0}\right)+x^{1}-x^{0}\right\| \leq \| \phi(x)-$ $\phi\left(x^{0}\right)\|+\| x^{1}-x^{0}\|\leq M \cdot\| x-x^{0}\|+\| x^{1}-x^{0} \| \leq$ $M \cdot r+(1-M) \cdot r=r$. Using theorem 3 we finish this proof.

\section{Discussion and conclusion}

Next we give some applications for theorem 6.

Conclusion 1. Let $f$ be a function. Using the translation the system of equations $f(x)=0$ in $\mathbb{R}^{n}$ is equivalent with the system of equations $x+f(x)=x$. We can consider the iterative function $\phi(x)=x+f(x)$. Now we apply theorem 6 for the iterative function $\phi$ and we obtain the following result for the function $f$ : if the function $f: B\left(x^{0}, r\right) \rightarrow \mathbb{R}^{n}$ is continuously differentiable function on $B\left(x^{0}, r\right)$, with $x^{0} \in \mathbb{R}^{n}$ and $r>0,\|1(x)+D(f)(x)\| \leq M<1$ for every $x \in B\left(x^{0}, r\right)$, where 1 is the identity application on $\mathbb{R}^{n}$ and $\left\|f\left(x^{0}\right)\right\| \leq(1-M) \cdot r$, then the system of equations $f(x)=0$ has a unique solution $x^{*} \in B\left(x^{0}, r\right)$ in the closed sphere $B\left(x^{0}, r\right) \subset \mathbb{R}^{n}$ and $x^{*}$ can be obtained as the limit of the sequence $\left\{x^{k}\right\}_{k \in \mathbb{N}}, x^{k+1}=x^{k}+f\left(x^{k}\right), k \in \mathbb{N}$.

Conclusion 2. Let $f$ be a function. Using the translation and the homothety the system of equations $f(x)=0$ is equivalent with the system of equations $x+\omega \cdot f(x)=x$, where $\omega \in \mathbb{R}^{*}$. We can consider the iterative function $\phi(x)=x+\omega \cdot f(x), \omega \in \mathbb{R}^{*}$. Now we apply theorem 6 for the iterative function $\phi$ and we obtain the following result for the function $f$ : if the function $f: B\left(x^{0}, r\right) \rightarrow \mathbb{R}^{n}$ is a continuously differentiable function on $B\left(x^{0}, r\right)$, with $x^{0} \in \mathbb{R}^{n}$ and $r>0,\|1(x)+\omega \cdot D(f)(x)\| \leq M<1$ for every $x \in B\left(x^{0}, r\right)$, and $\left\|f\left(x^{0}\right)\right\| \leq(1-M) \cdot \frac{r}{|\omega|}$, then the system of equations $f(x)=0$ has a unique solution $x^{*} \in B\left(x^{0}, r\right)$ in the closed sphere $B\left(x^{0}, r\right) \subset \mathbb{R}^{n}$ and $x^{*}$ can be obtained as the limit of the sequence $\left\{x^{k}\right\}_{k \in \mathbb{N}}, x^{k+1}=x^{k}+\omega \cdot f\left(x^{k}\right), k \in \mathbb{N}$.

Conclusion 3. Let $f$ be a function. Using the Newton's transformation the system of equations $f(x)=$ 0 is equivalent with the system of equations $x-$ $\left(f^{\prime}(x)\right)^{-1} f(x)=x$, where we suppose that the determinant $\operatorname{det}\left(f^{\prime}(x)\right) \neq 0$. We can consider the iterative function $\phi(x)=x-\left(f^{\prime}(x)\right)^{-1} f(x)$, which is the real $n$ dimensional Newton's method. We denote with $f^{*}$ : 
$B\left(x^{0}, r\right) \rightarrow \mathbb{R}^{n}$ the function $f^{*}(x)=\left(f^{\prime}(x)\right)^{-1} f(x)$. Now we apply theorem 6 for the iterative function $\phi$ and we obtain the following result for the function $f:$ if the function $f: B\left(x^{0}, r\right) \rightarrow \mathbb{R}^{n}$ is continuously differentiable function on $B\left(x^{0}, r\right)$, with $x^{0} \in \mathbb{R}^{n}$ and $r>0$, and $\operatorname{det}\left(f^{\prime}(x)\right) \neq 0$ for every $x \in B\left(x^{0}, r\right)$, and $\left\|1(x)-D\left(f^{*}\right)(x)\right\| \leq M<1$ for every $x \in$ $B\left(x^{0}, r\right)$, and $\left\|f^{*}\left(x^{0}\right)\right\|=||\left(f^{\prime}\left(x^{0}\right)\right)^{-1} f\left(x^{0}\right)|| \leq$ $(1-M) \cdot r$, then the real system of equations $f(x)=0$ has a unique solution $x^{*} \in B\left(x^{0}, r\right)$ in the closed disc $B\left(x^{0}, r\right) \subset \mathbb{R}^{n}$ and $x^{*}$ can be obtained as the limit of the sequence $\left\{x^{k}\right\}_{k \in \mathbb{N}}$, given by the real $n$ dimensional Newton's iterative formula: $x^{k+1}=x^{k}-$ $\left(f^{\prime}\left(x^{k}\right)\right)^{-1} f\left(x^{k}\right), k \in \mathbb{N}$.

Conclusion 4. Let f be a function. Using the transformation of the parallel method the system of equations $f(x)=0$ is equivalent with the system of equations $x-\frac{1}{\lambda} \cdot f(x)=x$, where $\lambda \in \mathbb{R}^{*}$. We can consider the iterative function $\phi(x)=x-\frac{1}{\lambda} \cdot f(x), \lambda \in \mathbb{R}^{*}, a$ variant of the parallel method in the space $\mathbb{R}^{n}$. Now we apply theorem 6 for the iterative function $\phi$ and we obtain the following result for the function $f$ : if the function $f: B\left(x^{0}, r\right) \rightarrow \mathbb{R}^{n}$ is a continuously differentiable function on $B\left(x^{0}, r\right)$, with $x^{0} \in \mathbb{R}^{n}$ and $r>0,\left\|1(x)-\frac{1}{\lambda} \cdot D(f)(x)\right\| \leq M<1$ for every $x \in$ $B\left(x^{0}, r\right)$, and $\left\|f\left(x^{0}\right)\right\| \leq(1-M) \cdot r \cdot|\lambda|$, then the system of equations $f(x)=0$ has a unique solution $x^{*} \in$ $B\left(x^{0}, r\right)$ in the closed sphere $B\left(x^{0}, r\right) \subset \mathbb{R}^{n}$ and $x^{*}$ can be obtained as the limit of the sequence $\left\{x^{k}\right\}_{k \in \mathbb{N}}$, given by the real $n$ dimensional iterative formula of the parallel method: $x^{k+1}=x^{k}-\frac{1}{\lambda} \cdot f\left(x^{k}\right), k \in \mathbb{N}$.

Conclusion 5. Let $f$ be a function. We build the real $n$ dimensional chord method in the following way: for the fixed $a=\left(a_{1}, a_{2}, \ldots, a_{n}\right) \in \mathbb{R}^{n}$ and for $i, j=$ $\overline{1, n}$ let us consider the functions $g_{i j}: B\left(x^{0}, r\right) \rightarrow$ $\mathbb{R}, g_{i j}(x)=\frac{f_{i}(x)-f_{i}(a)}{x_{j}-a_{j}}$, and the matrix type function $g(x)=\left(g_{i j}(x)\right)_{i, j=\overline{1, n}}$, where $x_{j} \neq a_{j}$ for every $j=\overline{1, n}$. Also we suppose that the determinant $\operatorname{det}(g(x)) \neq 0$. Using the transformation of chord method, the system of equations $f(x)=0$ is equivalent with the system of equations $x-(g(x))^{-1} \cdot f(x)=$ $x$. We denote with $g^{*}: B\left(x^{0}, r\right) \rightarrow \mathbb{R}^{n}$ the function $g^{*}(x)=(g(x))^{-1} \cdot f(x)$. We can consider the iterative function $\phi(x)=x-(g(x))^{-1} \cdot f(x)=x-g^{*}(x)$ for the real $n$ dimensional variant of the chord method. Now we apply theorem 6 for the iterative function $\phi$ and we obtain the following result for the function $f$ : if the function $f: B\left(x^{0}, r\right) \rightarrow \mathbb{R}^{n}$ is a continuously differentiable function on $B\left(x^{0}, r\right)$, with $x^{0} \in \mathbb{R}^{n}$ and $r>0$, and $\operatorname{det}(g(x)) \neq 0$ for every $x \in B\left(x^{0}, r\right)$ $\left\|1(x)-D\left(g^{*}\right)(x)\right\| \leq M<1$ for every $x \in B\left(x^{0}, r\right)$ and $\left\|g^{*}\left(x^{0}\right)\right\|=\left\|\left(g\left(x^{0}\right)\right)^{-1} \cdot f\left(x^{0}\right)\right\| \leq(1-M) \cdot r$, then the system of equations $f(x)=0$ has a unique solution $x^{*} \in B\left(x^{0}, r\right)$ in the closed disc $B\left(x^{0}, r\right) \subset \mathbb{R}^{n}$ and $x^{*}$ can be obtained as the limit of the sequence $\left\{x^{k}\right\}_{k \in \mathbb{N}}$, given by the iterative formula of the real $n$ dimensional chord method: $x^{k+1}=x^{k}-\left(g\left(x^{k}\right)\right)^{-1}$. $f\left(x^{k}\right), k \in \mathbb{N}$.

Conclusion 6. Let $f$ be a function. We build the real $n$ dimensional Steffensen's method in the following way: for every $i, j=\overline{1, n}$ let us consider the functions $h_{i j}: B\left(x^{0}, r\right) \rightarrow \mathbb{R}, h_{i j}(x)=\frac{f_{i}(x+f(x))-f_{i}(x)}{f_{j}(x)}$, and the matrix type function $h(x)=\left(h_{i j}(x)\right)_{i, j=\overline{1, n}}$, where $f_{j}(x) \neq 0$ for every $j=\overline{1, n}$. Also we suppose that the determinant $\operatorname{det}(h(x)) \neq 0$. Using the transformation of Steffensen's method, the system of equations $f(x)=0$ is equivalent with the system of equations $x-(h(x))^{-1} \cdot f(x)=x$. We denote with $h^{*}: B\left(x^{0}, r\right) \rightarrow \mathbb{R}^{n}$ the function $h^{*}(x)=$ $(h(x))^{-1} \cdot f(x)$. We can consider the iterative function $\phi(x)=x-(h(x))^{-1} \cdot f(x)$, the real $n$ dimensional variant of the Steffensen's method. Now we apply theorem 6 for the iterative function $\phi$ and we obtain the following result for the function $f:$ if the function $f: B\left(x^{0}, r\right) \rightarrow \mathbb{R}^{n}$ is a continuously differentiable function on $B\left(x^{0}, r\right)$, with $x^{0} \in \mathbb{R}^{n}$ and $r>0$, and $\operatorname{det}(h(x)) \neq 0$ for every $x \in B\left(x^{0}, r\right)$, and $\left\|1(x)-D\left(h^{*}\right)(x)\right\| \leq M<1$ for every $x \in$ $B\left(x^{0}, r\right)$ and $\left\|h^{*}\left(x^{0}\right)\right\|=\left\|\left(h\left(x^{0}\right)\right)^{-1} \cdot f\left(x^{0}\right)\right\| \leq$ $(1-M) \cdot r$, then the system of equations $f(x)=0$ has a unique solution $x^{*} \in B\left(x^{0}, r\right)$ in the closed disc $B\left(x^{0}, r\right) \subset \mathbb{R}^{n}$ and $x^{*}$ can be obtained as the limit of the sequence $\left\{x^{k}\right\}_{k \in \mathbb{N}}$, given by the iterative formula of the real $n$ dimensional Steffensen's method: $x^{k+1}=x^{k}-\left(h\left(x^{k}\right)\right)^{-1} \cdot f\left(x^{k}\right), k \in \mathbb{N}$.

\section{References}

[1] E. A. Volkov, Numerical Methods, Publishing Company MIR, Moscow, 1986.

[2] Béla Finta, Curs de analiză numerică, Universitatea "Petru Maior", Tg. Mures, 2004.

[3] Béla Finta, The Complex Version of a Result for Real Iterative Functions, Scientific Bulletin of the University of Medicine, Pharmacy, Sciences and Technology of Tirgu Mures, vol. 15(XXXII), no. 2, 2018, pag. 25-27.

[4] J. Cl. Evard and F. Jafari, A Complex Rolle's Theorem, Amer. Math. Monthly 99(1992), 858861.

[5] Árpád Száz, A Cauchy Mean Value Theorem for Complex Functions, Math. Student 64(1995), 125-127.

[6] Wikipedia. 